\title{
SEMI-GROUPS OF OPERATORS AND THE WEIERSTRASS THEOREM
}

\author{
NELSON DUNFORD AND I. E. SEGAL
}

In this note we give a short proof of the generalization ${ }^{1}$ of $\mathrm{M}$. H. Stone's representation of groups of unitary operators in Hilbert space and show how it yields the theorem of Weierstrass on uniform approximation by polynomials. This classical result in turn yields fairly easily the more recent algebraic-topological formulation of the Weierstrass theorem as given by M. H. Stone for real algebras and by I. Gelfand and G. Šlov ${ }^{2}$ for complex algebras.

For $0 \leqq s<\infty$ let $T_{s}$ be a linear operation in the real or complex Banach space $X$ satisfying the conditions

$$
T_{s+t}=T_{s} T_{t}, \quad T_{0}=I, \quad\left|T_{s}\right| \leqq 1, \quad \lim _{s \rightarrow t} T_{s} x=T_{t} x, \quad x \in X .
$$

Let $A_{h}=h^{-1}\left[T_{h}-I\right]$ and $D(A)$ be the domain of definition of the operator $A x \equiv \lim _{h \rightarrow 0} A_{h} x$.

THEOREM 1. If $T_{s}, 0 \leqq s<\infty$, is a semi-group of operators in $X$ satisfying (1) then $D(A)$ is dense in $X$ and uniformly for $s$ in any finite interval we have

$$
T_{s} x=\lim _{h \rightarrow 0} e^{s A_{h}} x, \quad x \in X .
$$

Note that $x_{s} \equiv s^{-1} \int_{0}^{s} T_{u} x d u \rightarrow x$ and that

$$
A_{h} x_{s}=(s h)^{-1}\left[\int_{s}^{o+h} T_{u} x d u-\int_{0}^{h} T_{u} x d u\right] \rightarrow A_{8} x \text { as } h \rightarrow 0 .
$$

Thus $D(A)$ is dense in $X$. Since $\left|e^{s A_{h}}\right|=\left|e^{(s / h) r_{h} e^{-s / h}}\right| \leqq e^{s / h} e^{-s / h}=1$ it suffices to prove (2) for $x \in D(A)$. If $x \in D(A)$ we have

Received by the editors June 10, 1946.

${ }^{1}$ See I. Gelfand, C. R. (Doklady) Acad. Sci. URSS. vol. 25 (1939) pp. 713-718; M. Fukamiya, Proc. Imp. Acad. Tokyo vol. 16 (1940) pp. 262-265; E. Hille, Proc. Nat. Acad. Sci. U.S.A. vol. 28 (1942) pp. 175-178; E. Hille, ibid. pp. 421-424. The particular form of the theorem given here is that of $E$. Hille.

2 M. H. Stone, Trans. Amer. Math. Soc. vol. 41 (1937) pp. 375-481; I. Gelfand and G. Silov, Rec. Math. (Mat. Sbornik) N.S. vol. 9 (1941) pp. 25-39. Our result differs from theirs in applying to spaces which are locally compact, and to subalgebras which are not assumed to contain a unit. 


$$
\begin{aligned}
\left|T_{s} x-e^{s A_{h}} x\right| & =\left|\int_{0}^{s} \frac{d}{d u}\left(e^{(8-u) A_{h}} T_{u} x\right) d u\right| \\
& =\left|\int_{0}^{s} e^{(s-u) A_{h}} T_{u}\left(A x-A_{h} x\right) d u\right| \\
& \leqq s\left|A x-A_{h} x\right| \rightarrow 0, \quad \text { as } h \rightarrow 0,
\end{aligned}
$$

which completes the proof. ${ }^{3}$

There is an $n$-parameter analogue of Theorem 1 . Suppose that $E_{n}^{+}$ is the set of points $s=\left(s_{1}, \cdots, s_{n}\right)$ in Euclidean $n$-space whose coordinates $s_{i}$ are non-negative. Let $T_{s}, s \in E_{n}^{+}$, be a linear operator in $X$ satisfying (1). Let $h_{i}=(0, \cdots, 0, h, 0, \cdots, 0)$ be the vector in $E_{n}^{+}$with $h>0$ in the $i$ th place and zeros elsewhere. Let $A(h, i)=h^{-1}\left[T_{h_{i}}-I\right]$ and $D\left(A_{i}\right)$ be the domain of definition of the operator $A_{i} x=\lim _{h \rightarrow 0} A(h, i) x$.

TheOREM 2. If $T_{s}, s \in E_{n}^{+}$, is a semi-group of operators in $X$ satisfying (1) then $\bigcap_{i} D\left(A_{i}\right)$ is dense in $X$ and uniformly for $s$ in a bounded closed set we have

$$
T_{s} x=\lim _{h \rightarrow 0} \exp \left(s_{1} A(h, 1)+\cdots+s_{n} A(h, n)\right) x, \quad x \in X .
$$

Let $C$ be the cube $0 \leqq s_{i} \leqq \delta$ and $x_{\delta}=\delta^{-n} \int_{C} T_{u} x d u$. Then $x_{\delta} \rightarrow x$ as as $\delta \rightarrow 0$. Just as before it follows that $x_{\delta} \in \bigcap_{i} D\left(A_{i}\right)$ so that this set is dense in $X$. Since $\exp \left(\sum_{i} s_{i} A(h, i)\right)=\prod_{i} \exp \left(s_{i} A(h, i)\right)$ equation (3) follows from Theorem 1 .

THEOREM 3. Every real or complex continuous function $x(s)$ defined on a bounded closed subset $E$ of Euclidean space is the uniform limit of polynomials in the coordinates $s_{1}, \cdots, s_{n}$ of $s$.

Let $X$ be the Banach space of real or complex bounded uniformly continuous functions $x(\lambda)$ defined on $E_{n}(|x| \equiv \sup |x(\lambda)|)$. We may and shall assume that $E \subset E_{n}^{+}$and that the function $x(s)=x$ of the theorem is a point of $X$. For $s \in E_{n}^{+}$define $T_{s} x=x(\lambda+s)$. By (3) then we have uniformly for $\lambda \in E_{n}$ and $s \in E$

$$
x(\lambda+s)=\lim _{h \rightarrow 0} \sum_{\nu=0}^{\infty} \frac{1}{\nu !}\left(s_{1} A(h, 1)+\cdots+s_{n} A(h, n)\right) x .
$$

Since $A(h, i) x$ is independent of $s$ and the series $\sum_{\nu=0}^{\infty}$ converges uniformly for $s \in E$ the desired conclusion follows by placing $\lambda=0$.

${ }^{8}$ In case $T_{s}$ is continuous in $s$ in the uniform topology of operators, obvious modifications of the above proof show that $A$ is a bounded operator with $\left|A_{h}-A\right|$ $\rightarrow 0$ and $T_{\mathrm{s}}=e^{\mathrm{sA}}$. 
TheOREM 4. Let $\mathfrak{A}$ be a closed (in the topology $|x|=\sup |x(\gamma)|$ ) subalgebra of the Banach algebra $C(\Gamma)$ of all continuous (real or complex) functions on the locally compact space which vanish at infinity. ${ }^{4}$ In the case of the complex algebra, suppose also that if $x \in \mathfrak{A}$ then there exists $a y$ in $\mathfrak{A}$ such that $\bar{x}(\gamma)=y(\gamma) .^{5}$ If for every $\gamma_{1} \neq \gamma_{2}$ there is an $x \in \mathfrak{A}$ such that $x\left(\gamma_{1}\right) \neq 0, x\left(\gamma_{2}\right)=0$, then $\mathfrak{A}=C(\gamma)$.

To begin with, suppose that the algebra is real and that $\Gamma$ is compact. In view of the known form of the general linear functional on $C(\Gamma)$, it suffices to show that a regular countably additive numerically-valued set function $\mu$ on the Borel sets of $\Gamma$ is identically zero provided

$$
\int_{\Gamma} x(\gamma) d \mu(\gamma)=0, \quad x \in \mathfrak{A} .
$$

Let $\Gamma_{1}$ be $\Gamma$ with the neighborhood system defined for $\epsilon>0$ and $x_{i} \in \mathfrak{A}$ with $x_{i}\left(\gamma_{0}\right)=0$ by

$$
N\left(\gamma_{0}\right)=\left[\gamma|| x_{i}(\gamma) \mid<\epsilon, i=1, \cdots, k\right] .
$$

Such neighborhoods are, by the continuity of $x_{i}$, open in $\Gamma$ and hence $\Gamma_{1}$ is compact. By hypothesis these neighborhoods separate points and since a continuous 1-1 map between compact spaces is a homeomorphism, we see that the neighborhoods $N\left(\gamma_{0}\right)$ form a basis for $\Gamma$. Let $F(s)=F\left(s_{1}, \cdots, s_{n}\right)$ be a bounded uniformly continuous function defined for $s=\left(s_{1}, \cdots, s_{n}\right) \in E_{n}$. Let $x_{1}, \cdots, x_{n}$ be elements of $\mathfrak{A}$. If $F\left(x_{1}, \cdots, x_{n}\right)$ is defined by the equation

$$
F\left(x_{1}, \cdots, x_{n}\right)(\gamma)=F\left(x_{1}(\gamma), \cdots, x_{n}(\gamma)\right), \quad \gamma \in \Gamma,
$$

it is plain that $F\left(x_{1}, \cdots, x_{n}\right) \in C(\Gamma)$. If $F(s)$ coincides with a polynomial (with $F(0)=0)$ in $s_{1}, \cdots, s_{n}$ on the set $\left(x_{1}(\Gamma), \cdots, x_{n}(\Gamma)\right.$ ), then clearly $F\left(x_{1}, \cdots, x_{n}\right) \in \mathfrak{A}$. But since $\mathfrak{A}$ is closed, Theorem 3 shows that $F\left(x_{1}, \cdots, x_{n}\right) \in \mathfrak{P}$ for an arbitrary continuous function $F(s)$ such that $F(0)=0$. Let $N$ be the neighborhood in $E_{n}$ defined by the inequalities $\left|s_{i}\right|<\epsilon, i=1, \cdots, n$, and let $\Phi(s)$ be the characteristic function of its complement. Clearly $\left(x_{1}(\gamma), \cdots, x_{n}(\gamma)\right) \in N$ if and only if $\gamma$ is in the neighborhood $N\left(\gamma_{0}\right)$ given by (5). Let $F_{\nu}(s)$ be a uniformly bounded sequence of continuous functions such that $F_{\nu}(s) \rightarrow \Phi(s), \quad s \in E_{n}$. Then $F_{\nu}\left(x_{1}, \cdots, x_{n}\right)(\gamma)$ approaches 1 for $\gamma \notin N\left(\gamma_{0}\right)$ and 0 for $\gamma \in N\left(\gamma_{0}\right)$ and so

${ }^{4}$ A function $x$ vanishes at infinity if for $\epsilon>0$ there is a compact set outside of which $|x(\gamma)|<\epsilon$.

${ }^{5} \bar{x}(\gamma)$ is the complex conjugate of $x(\gamma)$. 


$$
\mu\left(\Gamma-N\left(\gamma_{0}\right)\right)=\lim _{\nu} \int_{\Gamma} F_{\nu}\left(x_{1}, \cdots, x_{n}\right)(\gamma) d \mu(\gamma)=0,
$$

from which it readily follows that $\mu=0$.

In case $\mathfrak{A}$ is complex the proof is the same with the exception that $N\left(\gamma_{0}\right)$ is defined by

$$
N\left(\gamma_{0}\right)=\left[\left.\gamma|| x_{i}(\gamma)\right|^{2}<\epsilon, i=1,2, \cdots, n\right] .
$$

The proof for the case of $\Gamma$ locally compact and not compact can be reduced to the case of $\Gamma$ compact by compactification with a single point and addition of the constants to $\mathfrak{A}$, with removal of the point and the constants after the result just obtained has been applied.

YALE UNIVERSITY AND

INSTITUTE FOR ADVANCED STUdY 